\title{
Examination of Glass-Fibre Reinforced Composite Dental Fillings
}

\author{
Levente BORHY, ${ }^{1}$ Péter Zoltán FARKAS, ${ }^{2}$ András VOLOM ${ }^{3}$ \\ ${ }^{1}$ Budapest University of Technology and Economics, Faculty of Mechanical Engineering, Department of \\ Materials Science and Engineering, Budapest, Hungary, borhy.levente@edu.bme.hu \\ ${ }^{2}$ Dr. Volom Aesthetic Dentistry, Budapest, Hungary, farkas.peter013@gmail.com \\ ${ }^{3}$ Dr. Volom Aesthetic Dentistry, Budapest, Hungary, drvolom@drvolomdental.hu
}

\begin{abstract}
In dentistry, the use of dental fillings is a routine procedure. The use of fillings is a cheap, simple and lowharm dental operation, however, the filling of deep cavities is a difficult task. During this research, three types of fillings were tested: composite fillings bonded directly to the cavity walls, fillings bonded to the cavity walls with a semi-direct method, and composite fillings bonded to the cavity lined with polyethylene fibres. In the course of our examinations, the gaps between the wall of the dental cavity and the dental filling were observed using scanning electron microscopy. The results of these measurements can be used to determine the quality of each type of filling procedure.
\end{abstract}

Keywords: composite dental fillings, microstructural measurements, biomimetics.

\section{Introduction}

Dental diseases affect approximately 3,5 billion people worldwide. Based on the statistics of the WHO, nearly $100 \%$ of adults suffer from tooth decay, which is significant [1]. Therefore, restoring carious teeth is a very important public health issue. The methodology and material science behind the restoration is accordingly a dynamically evolving field of dentistry.

The main part of the tooth, from the neck to the root, is the dentin. Dentin is a hard but flexible organic tissue covered with enamel on the coronal part and dental cement on the root part. Dentin provides flexible and strong support for a much harder, more brittle enamel with little organic content [2]. A thin layer can be observed between the dentin and the enamel called the dentin enamel junction (DEJ), which ensures adhesion between the two tissues [3].

\subsection{Biomimetics}

Biomimetics is an innovative, multidisciplinary approach based primarily on collaboration between biology and engineering or other sciences, with the aim of developing solutions to a variety of practical problems by modeling biological systems [4]. In dentistry, the objective of biomimetics is to restore tissues, by combining methods and materials while keeping the original structure of the tooth, biomechanically and aesthetically [5-7]. One of the key objectives of the biomimetic approach in dentistry is maximizing the bonding force between the remaining tooth and the restoration (eg filling) during the binding of the restoration and reducing stress within the system formed by the tooth and the restoration to increase durability. Different tissues of the tooth have different bondability, which means that the strength of each cohesive/adhesive bond varies. Therefore, the bonding of the enamel and the dentin should be treated differently. The usage of a so-called hybrid layer for strong bonding is necessary, which is a reinforced transition layer infiltrated with a hydrophilic resin of the collagen fibres of demineralised dentin. It is important for the hybrid layer to decouple with time, otherwise the bond will not be strong enough and separation will occur on this surface [8, 9].

The different binding capacity of the tissues can cause polymerisation shrinkage of the composite dental fillings (if the composite is polymerized within the tooth with bulk fill method), which 
must be avoided to prevent the formation of gaps and cavities. Therefore, the reduction of the polymerisation shrinkage is key to a long-term success [10]. One possible method for achieving this is the application of dental fibre reinforcement, which reduces the risk of separation, by absorbing the stress caused by shrinkage [11]. Another possible solution is Semi-Direct dentin replacement, where the polymerisation takes place outside of the tooth, which is advantageous because the hybrid layer is not subjected to direct tensile stress due to the polymerisation shrinkage of the composite filling, and the hybrid layer has enough time to develop a stronger bond [12].

In dentistry, the configuration factor (or C-factor) refers to the number of bonded surfaces in an adhesive dental restoration. The bigger this value is, (which means more binding surface), the more polymerisation shrinkage will occur. During our research, we examined Class I cavities with a C-factor of 5 , which is highly exposed to cracks and residual stress. These cavities are conventionally filled in 2-3 layers which creates large internal stress and can lead to crack formation and propagation [13, 14].

Nowadays, dental fillings are mainly reviewed with microstructural and micro-tensile bond strength tests [15-17]. In this research, innovative dental fillings have been examined, which might substitute the usage of dental crowns in most cases, keeping most of the natural part of the tooth during the procedure.

Our goal is to create more flexible, gapless dental fillings than ever before with a novel dentin-like material and biomimetic method.

\section{Materials and methods}

\subsection{Materials}

In this study, Class I cavities were restored with fillings. The restorations were created by the same dentist (F. P. Z.) in all cases.

As filling material, EverX Posterior glass fibre reinforced composite was used, which was developed to replace the dentin in deep dental fillings (Class I) with a large C-factor. For reinforcement, the specially woven Ribbond polyethylene bondable reinforcement ribbon was applied. The bonding material was the Kuraray Clearfil SE Protect two-component, 6th generation, self-etching adhesive in all cases. The coverage of the filling was ensured by the Estelite Asteria Syringe nano composite. The gap between the tooth and the polymerised filling material for the Semi-Direct method was filled with Renamel Microfill liquid composite.

\subsection{Filling methods}

For the studies, structurally sound wisdom teeth were used, removed for prevention. The removed teeth were placed in room temperature 0,5\% Chloramine $\mathrm{T}$ solution for at least 24 hours before and after the preparation as well, in order to keep them moist, thus keeping the structure as intact as possible. During the teeth preparation, $4 \times 4 \times 4$ $\mathrm{mm}$ size deep Class I cavities were drilled and were preconditioned uniformly. Then, in all cases one of the three procedures were followed, thus creating the three groups for testing. The first group contained EverX bulk filled (Direct) fillings, where the composite was filled in the cavity in one load. The second group was the Semi-Direct method, where the bulk filled composites were polymerised extra-orally and then placed into the cavity. In the third group, polyethylene fibre reinforcement was used as underlining before the bulk filling.

\subsection{Measurement methods}

The restored wisdom teeth were prepared by the following method. The teeth were embedded in $\emptyset 20 \mathrm{~mm}$ silicone cylinders with Duracryl Plus two-component epoxy resin for further examination. After crosslinking, the obtained block was cut in the longitudinal direction with a Buehler IsoMet 1000 cutting machine at $2001 / \mathrm{min}$ speed with a diamond disc, with the lowest possible load. The cut sections were grinded with P600 and P1200 grit sandpaper, finally polished with a liquid containing $3 \mu \mathrm{m}$ diamond particles.

The samples were first examined at a lower magnification with a Zeiss EVO MA10 scanning electron microscope (SEM) at $20 \mathrm{kV}$ accelerating voltage to find and measure the gaps between the filling and the tooth. The resulting layers were examined at higher magnification. Since the resin and the tooth have low electrical conductivity, the surface was coated with a thin layer of gold to ensure conductivity.

\section{Results}

On the micrographs (Figure 1) the gap between the filling and the tooth and, its size can be observed.

As seen, the bulk filled EverX composite separated at the corners because of the stress concentration at the filling-tooth connection. This phenomenon gives an opportunity for further cavity formation and postoperative sensitivity. 

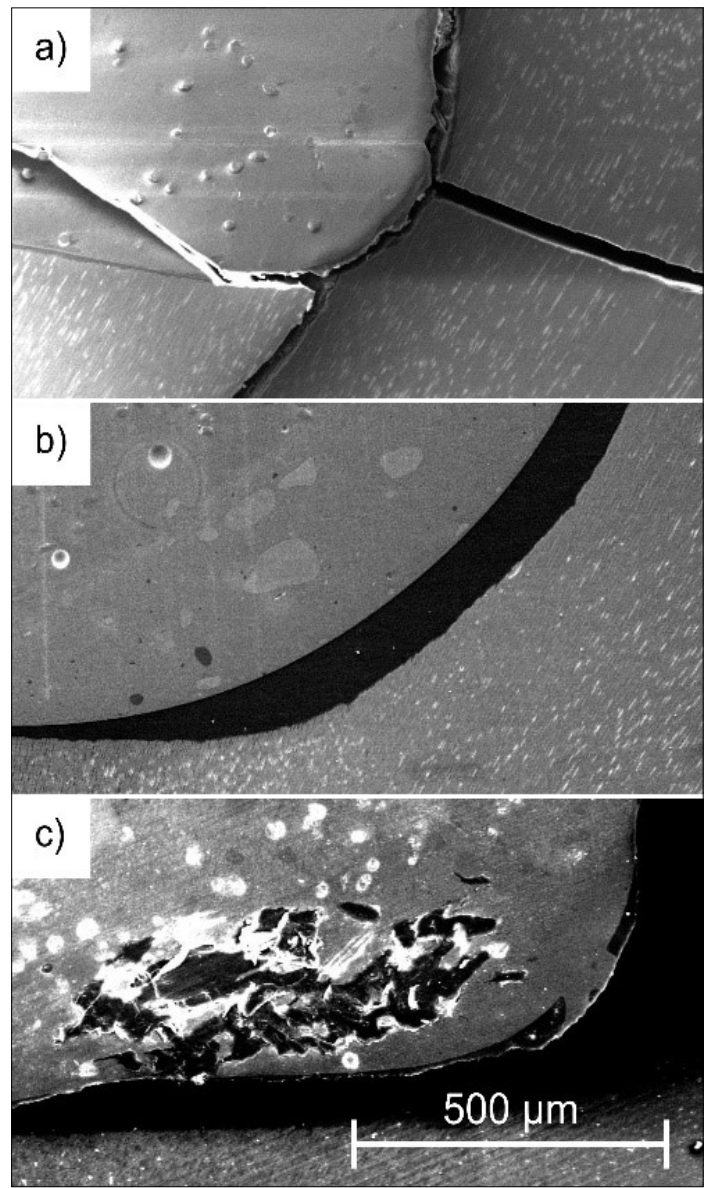

Figure 1. SEM micrographs of the direct (a), Semi-Direct (b) and Ribbond reinforced (c) dental fillings.

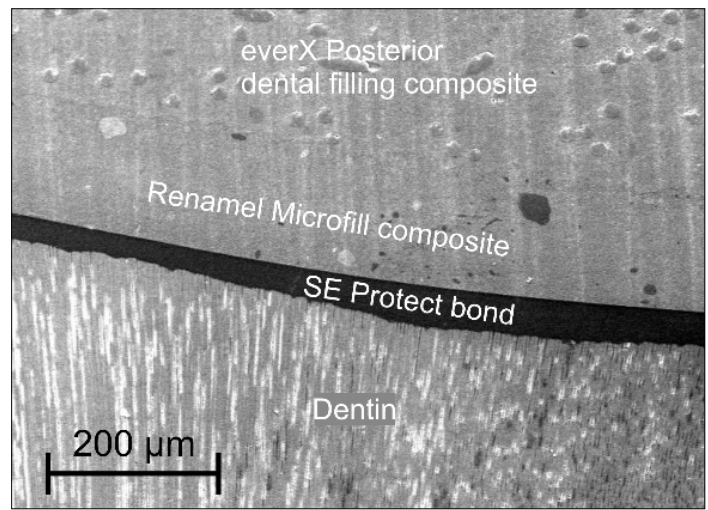

Figure 2. Layers of the semi-direct filling on SEM.

It can also be observed that the different layers of the Semi-Direct fillings are separable (Figure 2), and no gap can be found throughout the whole plane.
When the filling was reinforced with a Ribbond polyethylene fibre (Figure 1.c ), during sample preparation, the tooth itself cracked, also minor gaps were observed at the corner of the filling, which led to the conclusion that however the polyethylene fibre absorbed some stress caused by the shrinkage, some gap formation still occurs.

\section{Conclusions}

Based on microstructural studies, gap formation has been observed at the direct EverX filling, followed by a slight gap in the Ribbond strengthened sample. No gaps could be found on the sample with the Semi-Direct method filled tooth. Because the structure of every tooth differs, only a large number of samples could lead to a definite conclusion as to the differences between the filling methods.

The results reported here confirm the applicability of the test methodology and provide a basis for further, quantifiable studies. One of our future plans includes conducting micro-tensile bond strength tests, this way in addition to the qualitative data, we will have access to quantitative data suitable for statistical analysis on the adequacy of the restorations.

\section{Acknowledgement}

The publication of the work reported herein has been supported by the NTP-SZKOLL-20-0067 National Talent Programme of the Ministry of Human Capacities. The research reported in this paper and carried out at BME has been supported by the NRDI Fund (TKP2020 IES, Grant No. BME-IE-NAT; TKP2020 NC, Grant No. BME-NCS) based on the charter of bolster issued by the NRDI Office under the auspices of the Ministry for Innovation and Technology.

\section{References}

[1] GBD 2017 Disease and Injury Incidence and Prevalence Collaborators.: Global, regional, and national incidence, prevalence, and years lived with disability for 354 diseases and injuries for 195 countries and territories, 1990-2017: a systematic analysis for the Global Burden of Disease Study 2017, The Lancet, 392. (2018) 1789-1858. https://doi.org/10.1016/S0140-6736(18)32279-7

[2] Nanci A.: Chapter 8. Dentin-Pulp Complex. In: Ten Cate's Oral Histology (8. Ed.). Mosby, 2013. 165-204. https://doi.org/10.1016/B978-0-323-07846-7.00008-2

[3] Urabe I., Nakajima S., Sano H., Tagami J.: Physical properties of the dentin-enamel junction region. American Journal of Dentistry, 13. (2000) 129-135.

[4] ISO 18458: Biomimetics - Terminology, concepts and methodology, 2015. 
[5] Bazos P., Magne P.: Bio-emulation: biomimetically emulating nature utilizing a histoanatomic approach; structural analysis. The European Journal of Esthetic Dentistry, 6. (2011) 8-19.

[6] Alleman D. S., Nejad M. A., Alleman D. S.: The Protocols of Biomimetic Restorative Dentistry: 2002 to 2017. Inside Dentistry, 13. (2017) 1-6.

[7] Zafar M. S., Amin F., Fareed M. A., Ghabbani H., Riaz S., Khurshid Z., Kumar N.: Biomimetic Aspects of Restorative Dentistry Biomaterials. Biomimetics, 5. (2020) 34. https://doi.org/10.1016/B978-0-323-07846-7.00008-2

[8] Kemény A., Hajdu I., Károly D., Pammer D.: Osseointegration specified grit blasting parameters. Materials Today: Proceedings, 5/13. (2018) 26622-26627. https://doi.org/10.1016/j.matpr.2018.08.126

[9] van Meerbeek B., Yoshihara K., van Landuyt K., Yoshida Y., Peumans M.: From buonocore's pioneering acid-etch technique to self-adhering restoratlves. A status perspective of rapidly advancing dentai adheslve technology. The Journal of Adhesive Dentistry, 22. (2020) 7-34. https://doi.org/10.3290/j.jad.a43994

[10] Yadav R., Kumar M.: Dental restorative composite materials: A review. Journal of Oral Biosciences, 61. (2019) 78-83. https://doi.org/10.1016/j.job.2019.04.001

[11] Sadr A., Bakhtiari B., Hayashi J., Luong M. N., Chen Y. W., Chyz G., Chan D., Tagami J.: Effects of fiber reinforcement on adaptation and bond strength of a bulk-fill composite in deep preparations. Dental Materials, 36. (2020) 527-534. https://doi.org/10.1016/j.dental.2020.01.007
[12] Jayasooriya P. R., Pereira P. N. R., Nikaido T., Tagami J.: Efficacy of a resin coating on bond strengths of resin cement to dentin. Journal of Esthetic Restorative Dentistry, 15. (2003) 105-113. https://doi.org/10.1111/j.1708-8240.2003.tb00325.x

[13] Nikolaenko S. A., Lohbauer U., Roggendorf M., Petschelt A., Dasch W., Frankenberger R.: Influence of c-factor and layering technique on microtensile bond strength to dentin. Dental Materials, 20. (2004) 579-585. https://doi.org/10.1016/j.dental.2003.08.001

[14] Sakaguchi R. L., Peters M. C. R. B., Nelson S. R., Douglas W. H., Poort H. W.: Effects of polymerization contraction in composite restorations. Journal of Dentistry, 20. (1992) 178-182. https://doi.org/10.1016/0300-5712(92)90133-W

[15] Sano H., Chowdhury A. F. M. A., Saikaew P., Matsumoto M., Hoshika S., Yamauti M.: The micro-tensile bond strength test: Its historical background and application to bond testing. Japanese Dental Science Review, 56. (2020) 24-31. https://doi.org/10.1016/j.jdsr.2019.10.001

[16] Magne P., Ubaldini A. L. M.: Thermal and bioactive optimization of a unidose 3-step etchand-rinse dentin adhesive. The Journal of Prosthetic Dentistry, 124 (2020) 487.e1-487.e7. https://doi.org/10.1016/j.prosdent.2020.03.011

[17] Hayashi J., Espigares J., Takagaki T., Shimada Y., Tagami J., Numata T., Chan D., Sadr A.: Real-time in-depth imaging of gap formation in bulk-fill resin composites. Dental Materials, 35. (2019) 585-596. https://doi.org/10.1016/j.dental.2019.01.020 